\title{
Development of the theory of laying a hoisting rope on the drum of a mining hoisting machine
}

\author{
Kostiantyn Zabolotnyi ${ }^{1,{ }^{*}}$, Olena Panchenko ${ }^{1}$, and Oleksandr Zhupiiev ${ }^{1}$ \\ ${ }^{1}$ National Technical University "Dnipro Polytechnic", 49005, Dnipro, Dmytra Yavornytskoho Ave., \\ 19, Ukraine
}

\begin{abstract}
For new designs of mine hoisting machines, the development of scientifically-based solutions to increase the rope capacity and reduce the size of the machines is relevant. Based on the theory of laying the rope on a lined cylindrical drum developed by the authors, it is shown that when choosing a groove profile and winding pitch for a non-lined drum, it is necessary to use a geometric rope laying model, and for taking into account the elastic lining, it is necessary to use a physical model of static interaction of a rope with a scallop of the groove. It is recommended to use the U-tilda-groove profile, which will allow a $4.5 \%$ increase in the drum capacity while maintaining the rope's curvature radius that is permissible under safety rules during its installation.
\end{abstract}

\section{Introduction}

One of the scientific directions, which the department of mining machines and engineering is engaged in, is the optimization of the structures of mine hoisting machines (MHM) [1,2]. So when designing cylindrical drums of MHM, it was initially recommended to select the pitch of winding $t$ of the rope on the drum depending on the diameter of the rope $d_{k}$ by the formula [3]

$$
t=d_{k}+(2 \ldots 3) \mathrm{mm} \text {. }
$$

During operation of one of the first hoisting machines with a bicylindroconical drum of the $8 / 4.5 \times 2$, at deviation angles less than $0^{\circ} 49^{\prime} 30$ ", friction of the rope on the adjacent turn was discovered, which caused a strong crackle and vibration of the string. The operation of the hoisting installations was able to be resumed only when winding a rope of a smaller diameter (a decrease from 47.5 to $43.5 \mathrm{~mm}$ with a winding pitch of $50 \mathrm{~mm}$ ). It was necessary to determine the allowable pitch of winding the rope on the hoist drum of MHM.

In work [4], the authors attempted to answer this question by making the following assumptions: the turns of the rope are unrelated rings, the plane of which is perpendicular to the axis of the drum; the rope is wound on a drum on which there is no groove, but it also provides the possibility of winding with a given pitch; the flexural and transverse stiffnesses of the rope and lining, the string oscillations are ignored, and it is assumed that the rope is like a thin thread, until the contact with the drum lies in the plane of the angle of deviation.

*Corresponding author: mmf@ua.fm 
These assumptions allowed us to obtain an expression for the minimum distance between the points of adjacent ropes

$$
T(z)=\sqrt{\left(\sqrt{z^{2}+r_{b}^{2}}-r_{b}\right)^{2}+(t-z \cdot \operatorname{tg} \alpha)^{2}},
$$

where $z$ is the distance of an arbitrary point on the winding cable to the vertical plane passing through the axis of the drum; $r_{b}=0.5 d_{b}$ is the drum radius; $\alpha$ is the angle of deviation. For the selected value of the deviation angle and the diameter of the drum, graphs were constructed, from which the minimum distance between the ropes was determined, and then it was necessary to graphically find the required winding pitch from the specified minimum distance between the ropes.

When comparing the obtained results with the experimentally measured deviation angle and the winding pitch at which no intense wear of the ropes was observed, the authors made a generalization in two experiments: they recommended a negative gap $-(-1 \mathrm{~mm})$ between the ropes. Taking into account the negative clearance, the authors obtained the formula [3]

$$
t=1.025 \cdot d_{k}+3.6
$$

where $t$ and $d_{k}$ are expressed in mm.

The authors' reasoning error is that the axis of the rolled rope is not a straight line, since the rope is deflected by the ridge of the groove. The actual minimum distance between epy adjacent cables will be greater than that calculated using the proposed formula.

The adjustment of the formula for $t$ by introducing a negative gap was checked for the profile used for the wood lining. This profile is an arc of a circle with a radius $r_{d}$ approximately equal to the radius of the rope, and has sharp edges (further we will call this groove profile - U-profile)

Because of the lack of hard rocks, the manufacturing plants of the MHM refused to use the lining. The profile of the groove on the metal drum was not changed by the designers of the Donetsk Machine-Building Plant.

Initially, at Private Joint Stock Company "Novokramatorskyi Mashinobudivnyi Zavod" (PJSC "NKMZ"), the relationship between $t$ and $d_{k}$ by formula (1) was maintained for 5and 6-meter MHMs and a batch of such machines was produced. During operation, it turned out that in some cases, at large angles of deviation, friction of the laid rope with an adjacent one and its damage on sharp edges occurs. To prevent this, various technical solutions were used: a wooden lining was installed, the diameter of the hoisting rope was reduced, or more rope wear was deliberately allowed.

When creating new 5- and 6-meter MHMs, the designers suggested a groove cutting pitch, increased by 3-4 mm compared to the formula (3), a larger radius of chamfers and a profile consisting of two conjugate circular arcs (tilda-profile) with the radius of the bottom of the groove $r_{d}=0.5 t$ and the radius of the chamfer $r_{f}$, chosen so that the depth of the groove $h$ is equal to one third of the diameter of the rope. If the calculation by the formula (2) led to an increase in the winding pitch for machines with a drum diameter of $6 \mathrm{~m}$ by about $3 \mathrm{~mm}$, the transition to a new profile required a decrease in the rope capacity by increasing the winding pitch. For example, for a $63 \mathrm{~mm}$ rope, these pitches are 66, 68 and $71 \mathrm{~mm}$ respectively.

There was a technical contradiction - on the one hand, there are cases [4] of normal operation of MHM with a large drum diameter and groove cutting pitches recommended by formula (3), and, on the other hand, the tilda-profile recommended by PJSC "NKMZ" significantly reduces the cable intensity. To resolve this contradiction, it is necessary to develop a theory of laying the rope on the drum of mine hoisting machines on the basis of 
which the rational groove profiles should be proposed.

\section{Methods}

Figure 1 shows a diagram of the deviation of the rope from the deviation angle by the groove cut along a helix on the drum shell. For a reasonable choice of laying pitch $t$, it is necessary to analyze the relationship between the deviation angle $U_{d}$, the shape of the groove profile, its depth $h$, the rope diameter $d_{k}$, the drum diameter $d_{b}$ and the minimum distance to the adjacent rope, and for the lined drum, the account should be taken of the change in the groove profile because of lining compliance and there should be an assessment of the danger of its destruction by the rope, which is possible only by the finite element method in combination with the rope laying theory.

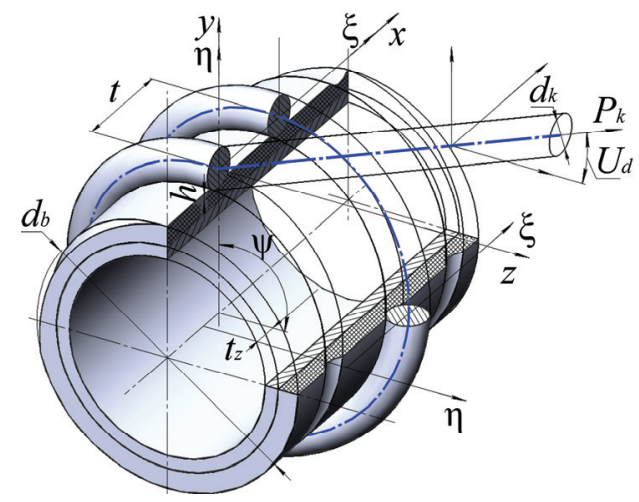

Fig. 1. The design scheme of laying the rope on the drum.

Suppose that at the point of descent of the rope from the drum (cable end point) (Fig. 1) the axial line (axis) of the rope is divided into two sections: a helix section on a cylindrical surface with a diameter $d_{b}$ and a straight line connecting the vanishing point and deflecting pulley, and a component with a normal to forming a cylinder angle $U_{d}$ (deviation angle). The plane touching the cylindrical surface and passing through this straight line is called the deviation plane. The minimum pitch of winding $t$ is the one at which the unwound (straight) and the adjacent lying coiled ropes touch, the distance between the axes of the ropes is equal to their diameter $d_{k}$.

We study the dependence of $t$ on $U_{d}, d_{b}$ and $d_{k}$. We introduce a local Cartesian coordinate system: centered at the vanishing point $O$, the axes $O x$ along the generator, $O y$ is the vertical, $O z$ is the horizontal and the cylindrical coordinate system connected with the cylindrical drum. The coordinates of any point on the axis of the rope lying on the helix are determined by the coordinates $t_{z}$ and $\psi$.

In the local coordinate system, the parametric system of equations of the helix:

$$
x=-\frac{t \cdot \psi}{2 \cdot \pi}, \quad y=0.5 \cdot d_{b} \cdot(\cos \psi-1), \quad z=0.5 \cdot d_{b} \cdot \sin \psi
$$

We expand expressions (4) in a Taylor series for small angles $\psi$ :

$$
x=-\frac{t \cdot z}{\pi \cdot d_{b}}, \quad y=-\frac{z^{2}}{d_{b}} .
$$

In the local coordinate system the equation of the unwound rope 


$$
x=z \cdot \operatorname{tg} U_{d}, \quad y=0,
$$

and the equation for the adjacent wound rope

$$
x=\frac{t \cdot z}{\pi \cdot d_{b}}, \quad y=-\frac{z^{2}}{d_{b}} .
$$

For a given $z$, we draw a plane orthogonal to the axis $O z$. The distance between the points of intersection of the axes of the rope in this plane

$$
T(z)=\sqrt{\left(\frac{z^{2}}{d_{b}}\right)^{2}+\left(z \cdot \operatorname{tg} U_{d}+\frac{t \cdot z}{\pi \cdot d_{b}}-t\right)^{2}} .
$$

Let us assume that the minimum value of $T_{\min }$ approximates the minimum distance between the axes of the ropes and the tangency of the ropes occurs at $T_{\min }=d_{k}$. The results of a numerical estimate of the minimum distance between the axes of the ropes and the obtained $T_{\min }$ show that, for real values of $t, U_{d}, d_{b}$ and $d_{k}$, the error does not exceed the accuracy of the calculation. Within the framework of this model, the task of finding the minimum laying pitch is formulated as the task of finding such a $t$ such that

$$
d_{k}=\inf _{z \in\left(0, d_{b} / 2\right)} T(z, t)
$$

Solving this problem numerically, we obtain the dependence of $t$ as a function of the three parameters

$$
t=f\left(U_{d}, d_{b}, d_{k}\right)
$$

Expression (10) is for a smooth drum of MHM. We take into account how $t$ changes depending on the profile of the groove. With each point of the helix with the coordinate $\psi$ we associate the center of the moving Cartesian coordinate system $\xi \cdot A \cdot \eta$, the axis $\xi$ of which is directed along the drum generator, and the axis $\eta$-along the radius.

For small deviation angles, it is permissible to consider the plane of this coordinate system to be orthogonal helix and write the equation of the groove profile in the form $\eta=F(\xi)$. The inverse function $\xi=f(\eta)$ exists for profile sections that do not contain straight line segments parallel to the $\xi$ axis. For a profile of a groove consisting of circular arcs (the radius of concave arcs is greater than the radius of the rope) and line segments tangent to them, you can build an equidistant line (centroid), the points of which are inside the groove and are at a distance equal to the radius of the rope from the groove profile, the equation of which is $\xi=g(\eta)$. The screw surface described by the centroid, which rotates evenly around the axis of the drum, simultaneously performs translational movement along the same axis with parametric equations

$$
x=-\frac{t \cdot z}{\pi \cdot d_{b}}+g(\eta), \quad y=\left(\eta+\frac{d_{b}}{2}\right) \cos \psi-\frac{d_{b}}{2}, \quad z=\left(\eta+\frac{d_{b}}{2}\right) \cdot \sin \psi,
$$

we call equidistant.

The axis of the rope from the vanishing point under the condition of a smooth (without friction) interaction of the rope with the groove consists of three sections: the geodesic line $B E$ on the equidistant and tangent to it segments of straight lines $O B$ and $E G$, where point $G$ 
belongs to the deflecting pulley.

Let us describe the algorithm for constructing the line $O B E G$. Let us suppose that this line is flat, lying in the plane of deviation. Then, going through the intersection point (let's call it deflecting) with a given pitch, equidistants with the deviation plane

$$
z=\frac{d_{b}}{2} \operatorname{tg} \psi, \quad \eta=\frac{d_{b}}{2}\left(\frac{1}{\cos \psi}-1\right), \quad x=-\frac{t \cdot z}{\pi \cdot d_{b}}+g(\eta)
$$

and calculating the derivative $d x / d z$ in each of them, we find the points $B_{0}$ and $E_{0}$ as points $O B_{0}$ and $E_{0} G$ to the deflecting point. For small angles, the deflection equation is converted to

$$
x=-\frac{t \cdot z}{\pi \cdot d_{b}}+g\left(\frac{z^{2}}{d_{b}}\right)
$$

and the coordinates of the point $B_{0}$ satisfy the equation

$$
x_{B}=\frac{d x}{d z} z_{B}=-\frac{t \cdot z_{B}}{\pi \cdot d_{b}}+\frac{d g}{d \eta} \cdot \frac{2 z_{B}^{2}}{d_{b}}=-\frac{t}{\pi} \sqrt{\frac{\eta_{B}}{d_{b}}}+g\left(\eta_{B}\right) .
$$

The coordinate $\eta_{B}$ is determined from the equation

$$
g\left(\eta_{B}\right)=2 \eta_{B} \frac{d g}{d \eta}\left(\eta_{B}\right)
$$

Similarly, to determine the coordinates of the point $E_{0}$, we obtain the equation

$$
2 \frac{d g}{d \eta}\left(\eta_{E}\right) \sqrt{\frac{\eta_{E}}{d_{b}}}=\operatorname{tg}\left(U_{d}\right)+\frac{t}{\pi \cdot d_{b}}
$$

Similar to formula (8) the minimum distance between the ropes corresponds to the minimum of the function

$$
R(z)=\sqrt{\left(x_{E}+\operatorname{tg}\left(U_{d}\right)\left(z-z_{E}\right)-t+\frac{t \cdot z}{\pi \cdot d_{b}}\right)^{2}+\frac{z^{4}}{d_{b}^{2}}}
$$

and is determined as the root of the cubic equation

$$
\frac{2 z^{3}}{d_{b}^{2}}+\left(x_{E}+\operatorname{tg}\left(U_{d}\right) \cdot\left(z-z_{E}\right)-t+\frac{t \cdot z}{\pi \cdot d_{b}}\right) \cdot\left(\operatorname{tg}\left(U_{d}\right)+\frac{t}{\pi \cdot d_{b}}\right)=0 .
$$

In order to apply the algorithm (9) of finding the minimum pitch, it is necessary to specify a function defining the groove profile $\xi=f(\eta)$ in the form $\xi=f(\eta, t)$. In other words, it is necessary to form a one-parameter family of the groove profile defined for any $t$ from a certain allowable interval.

Summarizing the shapes of the groove profiles used by PJSC "NKMZ", we represent it as a conjugation of arcs of circles and straight line segments $O^{\prime} P^{\prime} Q^{\prime} V^{\prime} W^{\prime}$ (Fig. 2). The centroid of $O P Q V W$ is also shown here. The main parameters of the profile $t, h, a, b, r_{f}, r_{c}$ and $\beta$ are related by the following ratios: 


$$
\begin{aligned}
& \left(r_{c}+r_{f}\right) \sin \beta+b \cdot \cos \beta+a=0.5 t \\
& \left(r_{c}+r_{f}\right)(1-\cos \beta)+b \cdot \sin \beta=h .
\end{aligned}
$$

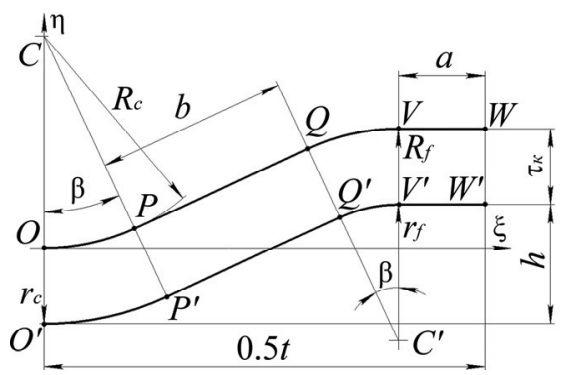

Fig. 2. The generalized form of groove profiles.

By specifying in some way the functions $h(t), a(t), b(t), r_{f}(t), r_{c}(t)$ and $\beta(t)$, satisfying constraints (17), one or another profile family is determined. Consider the following four families. First of all, the main one - U-profile. It is given the values $b=0, r_{f}=0, h$, and $r_{c}$. The following value is calculated

$$
\tau=\sqrt{r_{c}^{2}-\left(r_{c}-h\right)^{2}}
$$

If it turns out that $\tau>0.5 t$, it is assumed

$$
\tau=0.5 t ; \quad h=r_{c}-\sqrt{r_{c}^{2}-\tau^{2}},
$$

and then it is calculated

$$
a=0.5 t-\tau ; \quad \beta=\operatorname{arctg}\left(\frac{t-2 a}{2\left(r_{c}-h\right)}\right) .
$$

The tilda-profile is characterized by the dependence $r_{c}=0.5 \cdot t$ with $a=0, b=0$. Then from (17) it follows

$$
r_{f}=\frac{\left(r_{c}-h\right)^{2}}{2 h}, \quad \beta=\operatorname{arctg}\left(\frac{r_{c}}{r_{c}+r_{f}-h}\right) .
$$

To check the possibility of reducing the bending of the rope during installation, a Vprofile was introduced, for which $r_{f}=0$ and the values of $h$ and $\beta$ are set. From relations (17) it follows:

$$
b=\frac{t-2 r_{c} \cdot \sin \beta-a}{\cos \beta}, \quad h=r_{c}(1-\cos \beta)+b \sin \beta
$$

In the algorithm for finding $t$ for the $\mathrm{V}$-profile, it is provided that initially there was no flat section and the height of the ridge of the groove was unlimited. Only at the very end, when $t$ had already been found, the unused material was cut off and $h$ and $a$ were determined.

The U-profile was introduced for the lined drum, so there were no chamfers in it. To reduce rope wear during installation, the profile is proposed that occupies an intermediate 
position between the $\mathrm{U}$ - and tilda-profiles - the U-tilda-profile, characterized by an unequal zero given by $r_{f}$.

From the values of $h$ and $r_{c}$ is calculated

$$
\tau=\sqrt{\left(r_{c}+r_{f}\right)^{2}-\left(r_{c}+r_{f}-h\right)^{2}} .
$$

If it turns out that $\tau>0.5 \cdot t$, then it is accepted that

$$
\tau=0.5 t ; \quad h=r_{c}+r_{f}-\sqrt{\left(r_{c}+r_{f}\right)^{2}-\tau^{2}},
$$

and then $a=0.5 \cdot t-\tau$ is calculated.

After specifying the profile for each of the $O^{\prime} P^{\prime}, P^{\prime} Q^{\prime}, Q^{\prime} V^{\prime}, V^{\prime} W^{\prime}$ sections, the corresponding sections of the centroid, $O P, P Q, Q V, V W$ sections were constructed at a distance equal to the radius of the rope from the profile line. Then, for each section of the centroid the first and second derivatives in the moving coordinate system were calculated. By formulas (12), the transition to the construction of centroids in the deviation plane was carried out for each $z$, thereby constructing the entire equidistant.

Writing the equation of the tangent $E_{0} G$ in the form similar to (6), we find the distance $T_{\min }$ between it and the axis of the wound rope (7) using formulas similar to (8) and (9).

The above algorithm is implemented in the program [5]. Figures $3-6$ show the screen forms of this program for the four profile families described at the same deviation angle $\left(1.5^{\circ}\right)$ and the relationship of the drum diameter to the rope diameter (200) and the groove depth to the rope diameter $(0.3)$. The left part of the form shows the deviation plane, in which the $B E K$ line corresponds to the axis of the laid rope, $B E U$ line - to the equidistant section of the deviation plane (corresponding to the crest of the groove), $K S$ line is the geometric locus of the points of the deviation plane from the adjacent wound rope at a specified distance (clearance between the ropes was taken as zero, which corresponded to the specification of this distance equal to the diameter of the rope). Point $K$ is the point of contact between adjacent ropes. The right side of the screen form in the moving coordinate system shows the profile of the groove, the centroid and points $B$ and $E$ - the beginning and end of the contact of the laid rope with the ridge of the groove. In addition, from these points in the direction of the groove walls, lines of action of the resultant pressure forces of the rope on the comb are drawn. The values of the radius of curvature were calculated along the $B E$ line in the calculation. On screen form, all values, except the radii of curvature, are related to the diameter of the rope, and the radii of curvature - to the radius of the rope. The deviation angle is displayed in degrees. Points $P$ and $Q$ correspond to Figure 2. From a comparison of Figures $3-6$, it follows that the maximum rope spacing $(5 \%$ of the minimum) corresponds to the tilda-profile recommended by NKMZ, and the minimum to the previously used U-profile with sharp edges. The introduction of chamfers commensurate with the radius of the wire (U-tilda-profile in Figure 5) slightly increases the pitch $(0.4 \%$ of the minimum). The radii of curvature for all profiles do not exceed the limit values according to the Safety Rules.

Let us estimate the error of the assumptions of the above mathematical model, which we call the geometric theory of styling. To take into account the deviation from the deviation plane of the $B E$ section of the rope axis, we will construct a geodetic model (the program [5]). We divide the interval $B_{0} E_{0}$ into a given number of points and draw a plane through each of them and through the axis of the drum. We obtain a sequential set of sections of equidistant representing the above-described centroid shifted along the axis of the drum. 


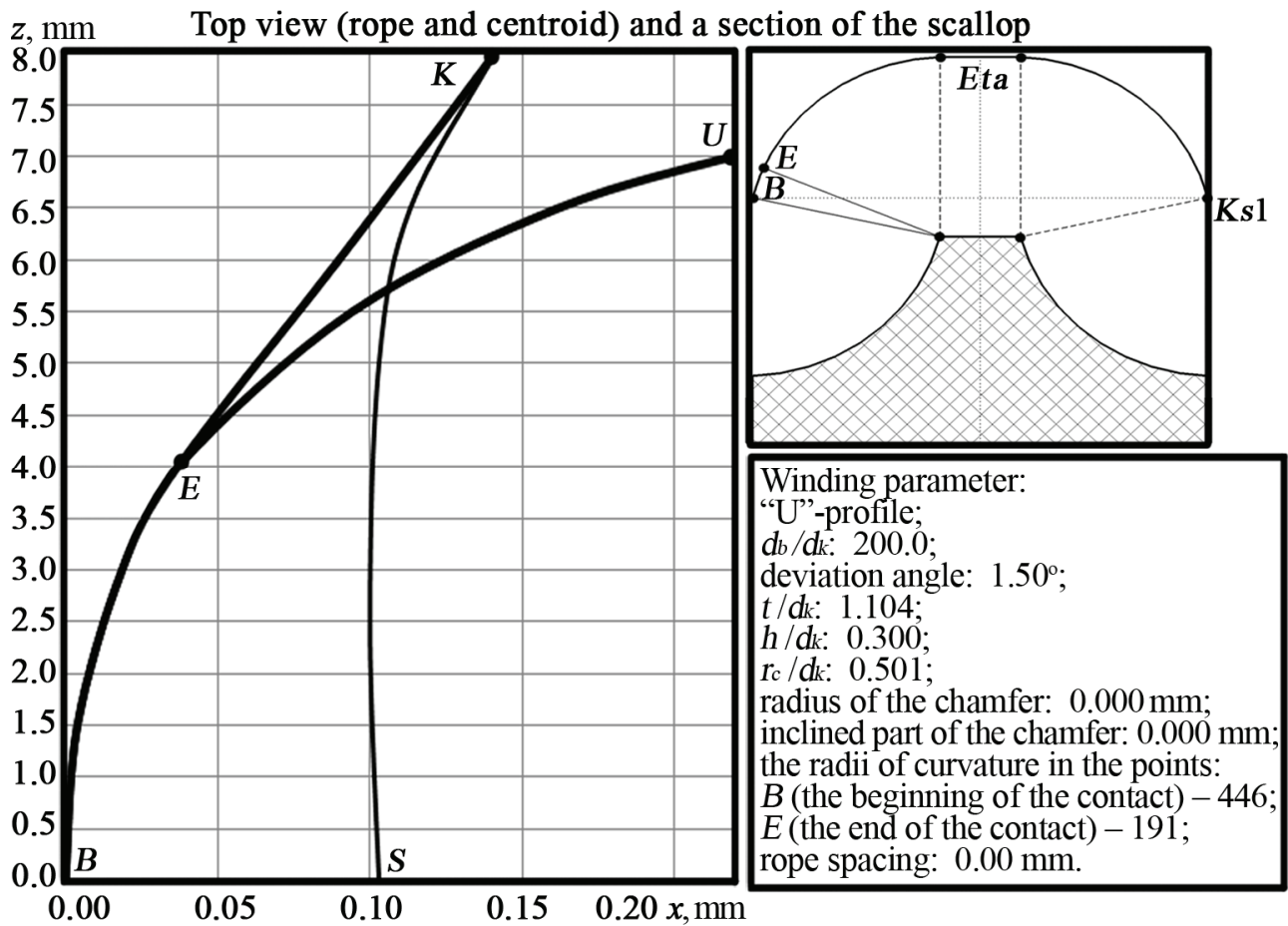

Fig. 3. Screen form of the program [5] for U-groove profile with sharp edges.

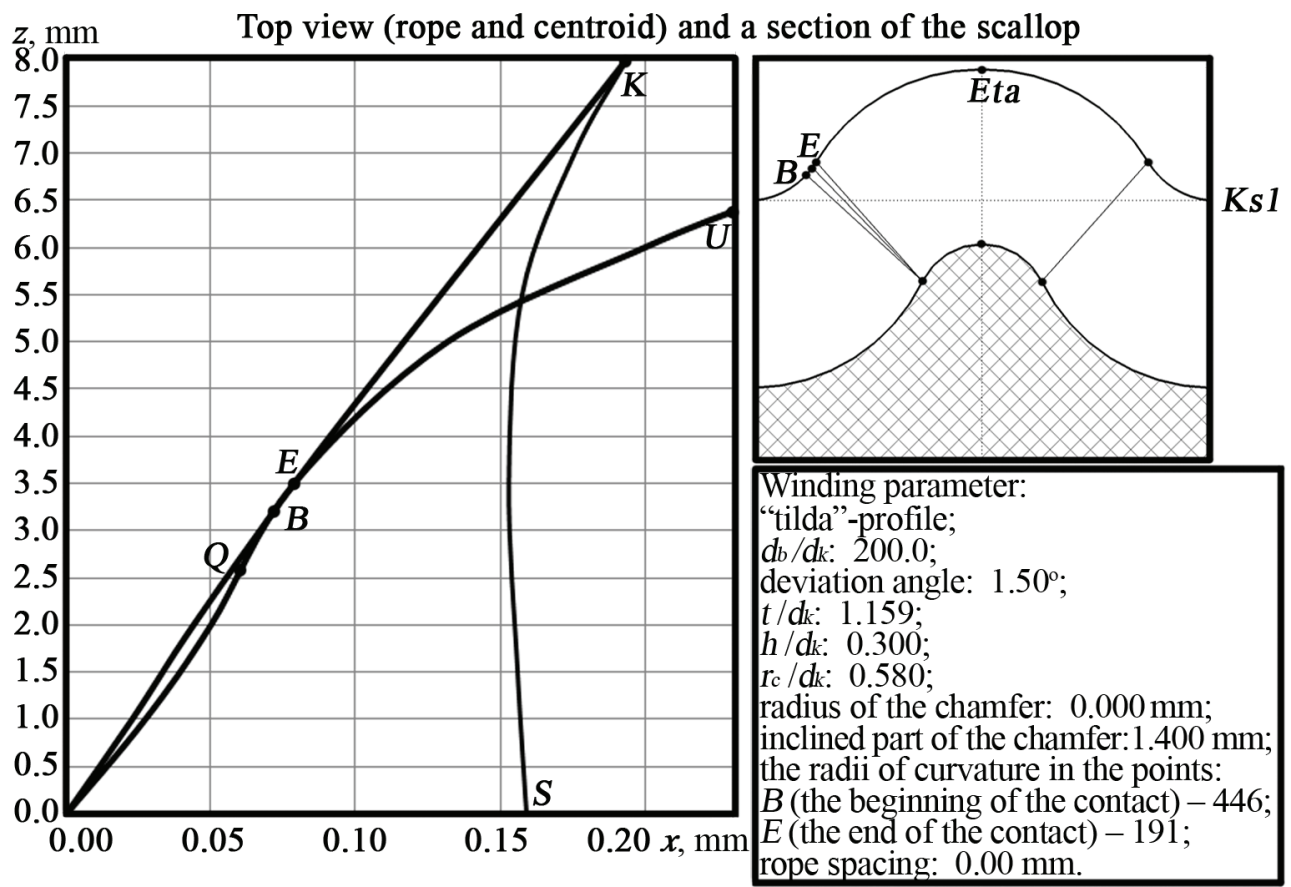

Fig. 4. The screen form of the program [5] for tilda-profile of the groove. 


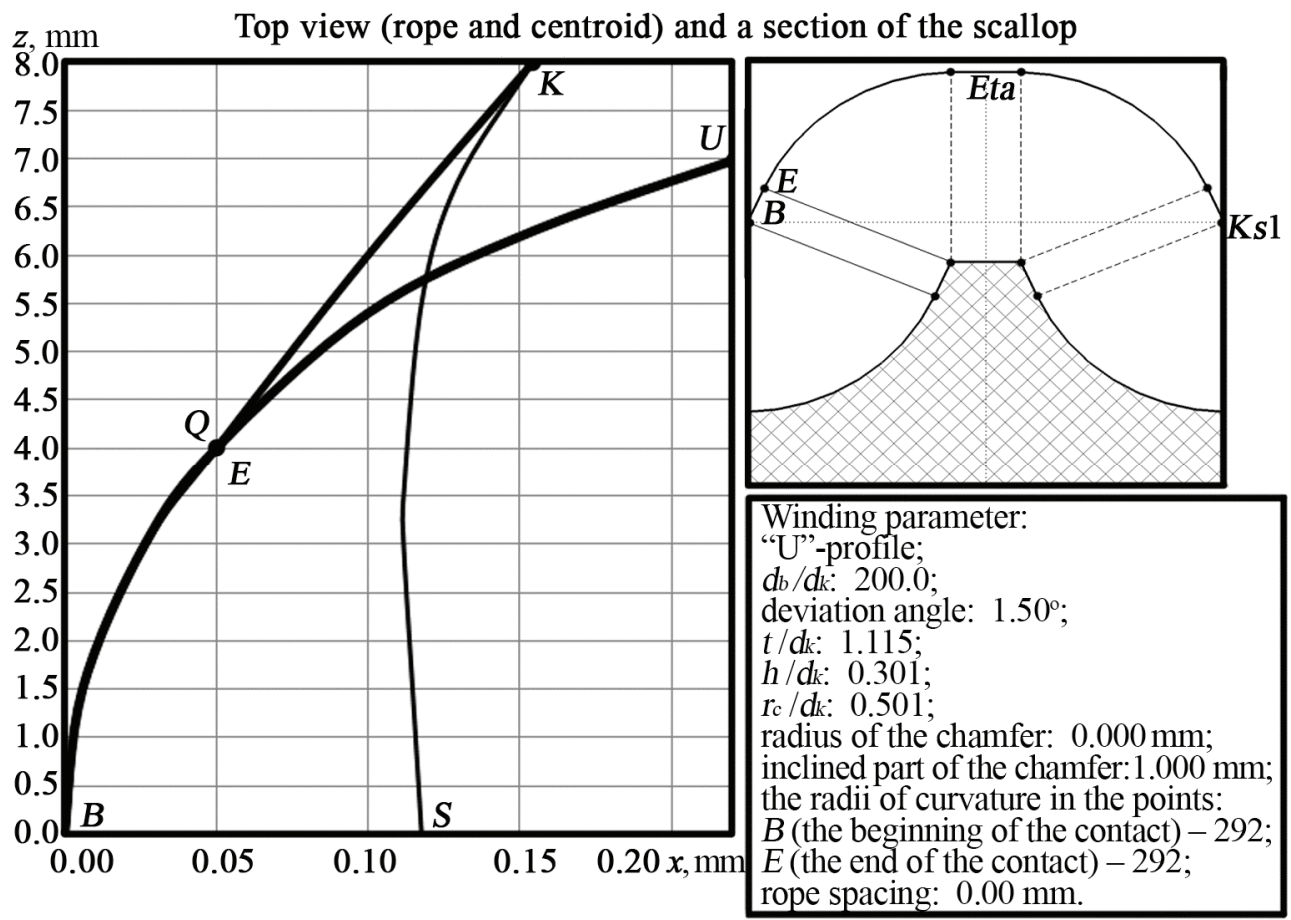

Fig. 5. Screen form of the program [5] for U-profile of the groove with chamfers.

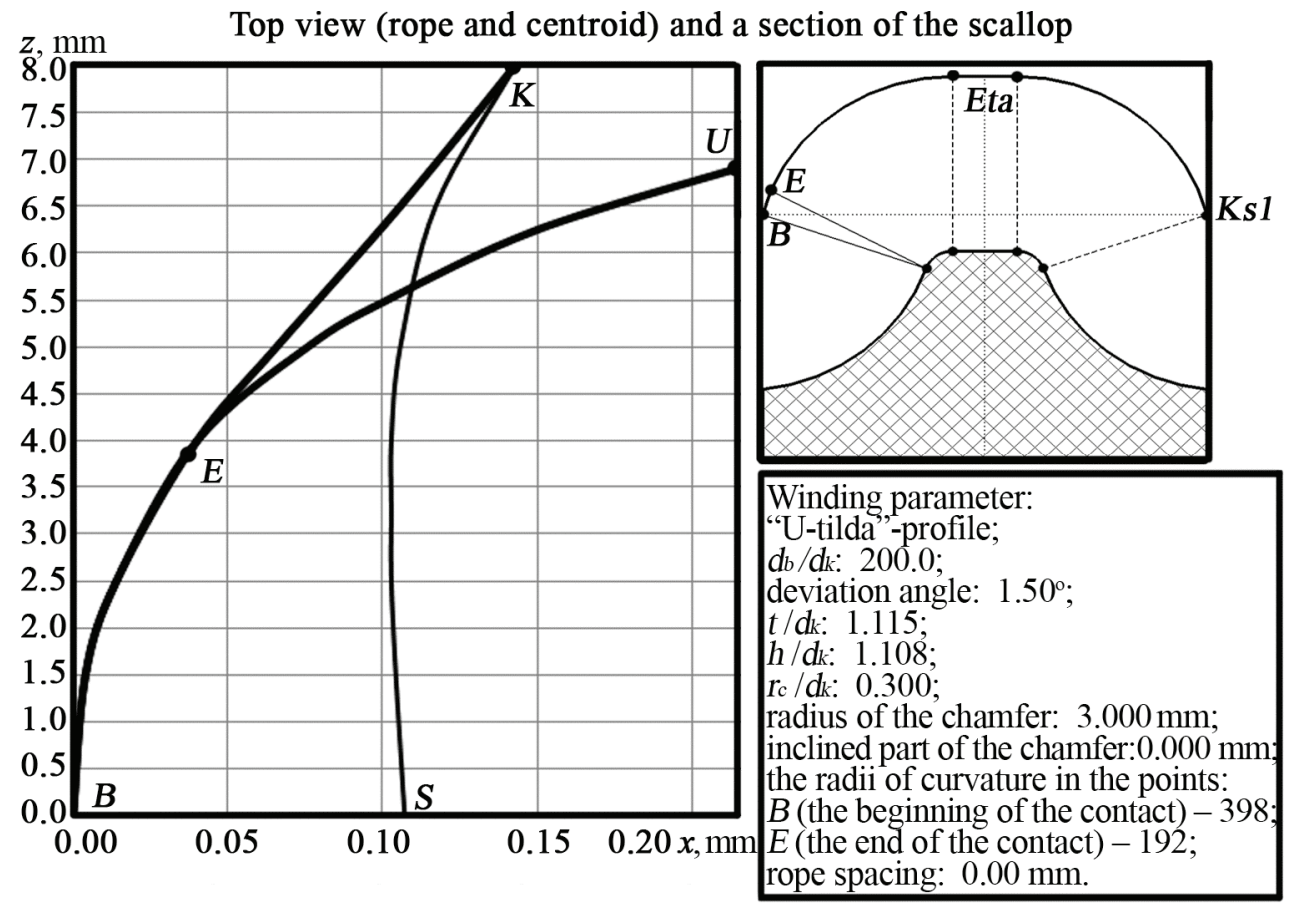

Fig. 6. Screen form of the program [5] for U-tilda-profile of the groove.

Let us consider the equilibrium of the system of material points on these lines, which are connected with each other and with the points $O, G$ with a stretched thread. To 
determine the boundary points of the contact area of points $B$ and $E$, we use an iterative algorithm. As the first approximation, let us specify the coordinates of the material points found from the geometric problem. Assuming that their configuration is determined by imposed connections in the section planes of equidistant, from the equilibrium equations we find the reactions of these bonds (normal and tangent to the centroid components).For points where the normal component is less than zero, we set the movement along the centroid, which is proportional to the tangent component. If in this iteration in one of the $B_{0}$ or $E_{0}$ boundary points the normal component is greater than zero (bond stretching), we will not specify the motion of this point, and as a new boundary point $B_{0}$ or $E_{0}$ we will define the neighboring point with $B_{0}$ or $E_{0}$ located inside the contact area this iteration. We repeat the iteration process until, with a given accuracy, we determine points $B$ and $E$ as the limit points of the sequence $B_{0}$ and $E_{0}$. To take into account the friction forces, limiting friction forces were introduced in the geodesic model, caused by the normal pressure at each point and directed upward in the cross section for the unwinding case and downward for laying the rope on the drum. In the same model, for taking into account the transverse rigidity of the rope, the material points were not located on the equidistant, but were connected by springs with a rigidity equivalent to the transverse rigidity of the rope, with the corresponding points of the groove profile. The rope was modeled by a massive stretched rod with a viscosity $\lambda$ :

$$
\begin{aligned}
& E I \frac{\partial^{4} u}{\partial z^{4}}-P_{k} \frac{\partial^{2} u}{\partial z^{2}}+\lambda \frac{\partial u}{\partial t}=F_{u}\left(u, v, \frac{\partial u}{\partial t}, \frac{\partial v}{\partial t}\right) \\
& E I \frac{\partial^{4} v}{\partial z^{4}}-P_{k} \frac{\partial^{2} v}{\partial z^{2}}+\lambda \frac{\partial v}{\partial t}=F_{v}\left(u, v, \frac{\partial u}{\partial t}, \frac{\partial v}{\partial t}\right)
\end{aligned}
$$

where $E I\left(P_{k}\right)$ is the flexural rigidity of the rope, stretched by the force $P_{k}, u$ and $v$ are the displacements of the rope points along the $x$ and $y$ axes, respectively (Fig. 1); and $F_{u}$ and $F_{v}$ are the projections on the same axes of forces in the springs simulating the lateral rigidity of the rope. In the case, if these efforts turned out to be tensing, they were equated to zero. For the solution, an iterative stable scheme was chosen, according to which the equations for the known forces in the springs were solved at each moment of time, which in turn were distinguished at the previous time moment by the given rope configurations and profile shapes. Similar to the GEONAM problem, at each iteration, the distance to the adjacent rope was calculated and the value of the laying pitch was chosen so that at the next moment of time this distance tended to zero.

The course of the iterative process was controlled by displaying graphic information about the shape of the rope and contact pressure forces on the display screen.

The error in determining $T_{\min }$ for the checked profiles for geometric and geodesic models is less than $0.5 \%$.

The developed laying theory allows to solve the problem of choosing a rational profile and laying pitch for each deviation angle. For this purpose, all the characteristics of the profile under study are represented as a function of the laying pitch $t$. When describing these functions, it is necessary to take into account the technological limitations (for example, the constancy of the depth of the groove or the radius of the bottom of the groove), as well as the introduction of additional characteristics (for example, straight line segments between the mating arcs) for large stacking pitch values.

The effect of the flexural rigidity of the rope and the amplitudes of the transverse oscillations of the string (the geometric model was used with the function $y=-z / d_{b}$ replaced by the function of a more complex form) was expressed in a decrease in $T_{\min }$ for the tested profiles by $0.9 \%$. 
When using a nonmetallic removable lining, due to the pressure of the hoisting rope, the shape of the scallop deviates significantly from the given groove profile, which should cause a decrease in $T_{\min }$ and, accordingly, an increase in $t$. The risk of destruction (cleavage) of the scallop should also be assessed. The model of the interaction of a rope with a lining (programs [5]) is proposed, based on the combination of geometric and geodesic models with the presentation of the lining as a body of the three-dimensional theory of elasticity and a rope as a stretched rod with flexural rigidity. The iterative process of the geodetic model [5] is applied, at each pitch of which the FEM elastic problem, developed by the authors by the complex [5], is solved. In the right side of equations (15), for $u\left(z_{i}\right) v\left(z_{i}\right)$, instead of the functions $u\left(z_{i}\right), v\left(z_{i}\right)$, the functions of long-range interaction were used $K\left(u\left(z_{i}\right), v\left(z_{i}\right), u\left(z_{i-1}\right), v\left(z_{i-1}\right), u\left(z_{i+1}\right), v\left(z_{i+1}\right), \ldots\right)$, which are determined from the Green matrix (matrices stiffness) obtained by numerical solution of the three-dimensional theory of elasticity. For the first approximation, the form of the rope obtained from the programs [5] was used.

In the area of the intended contact $B E$ (Fig. 3), defined by the programs [5], the lining comb was divided into 29 layers (Fig. 7), and each layer into two symmetrical curvilinear four-sided sections with the same number of nodes on each side (Fig. 8). The lateral compression of the rope was modeled by rods that do not resist bending (Fig. 9). Each top of the comb was connected by such rods with a corresponding rope (Fig. 10). In this case, it was assumed that the wound rope connections are rigidly fixed in space. In addition, the coiled rope was connected with the same rods to the bottom of the groove. In the course of the iterative solution of the problem of spatial contact theory of elasticity, stresses were checked in all rods and, where tensile forces occurred, the rod was excluded from the design scheme (since, as part of the stated contact task, ropes in the transverse direction can only experience compression).

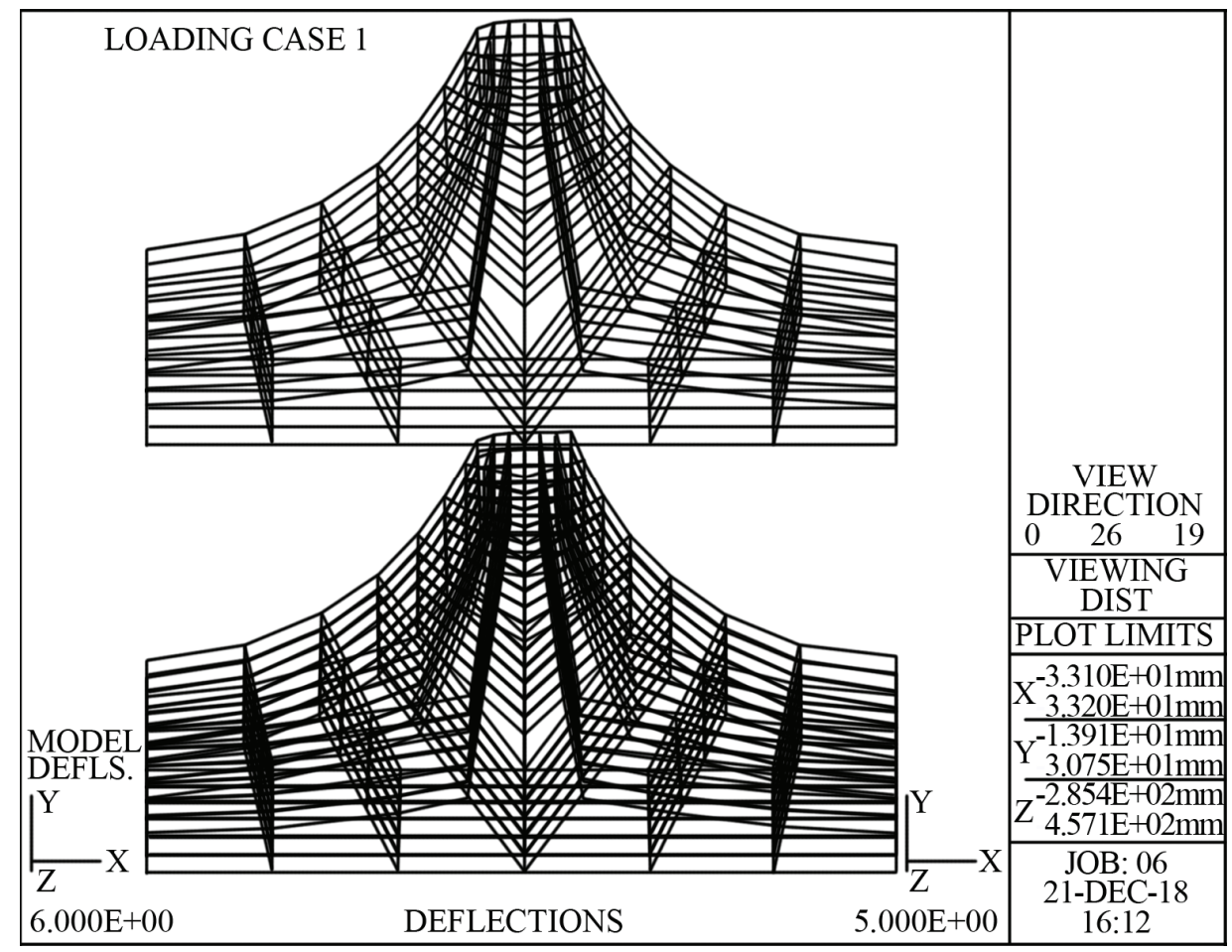

Fig. 7. Moving of the points in two layers of the scallop when winding the rope according to the model (screen form [5]). 


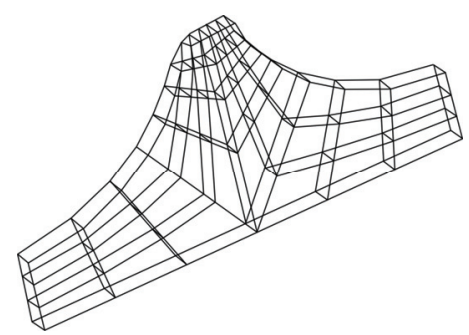

Fig. 8. Element-based breakdown of one layer (screen form).

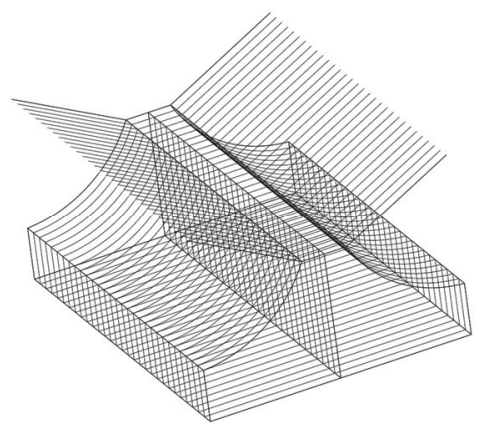

Fig. 9. Layered breakdown of scallops and rope elements.

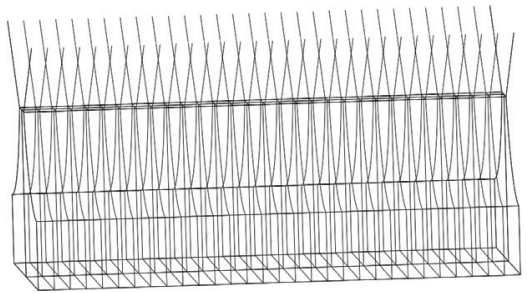

Fig. 10. Side view of the comb and rope (screen form).

After the end of the iterative process, the displacement of the shell points (Fig. 10) and the voltages in it (Fig. 11) were displayed in a tabular form on a solid carrier and on the monitor screen.

One more peculiarity of the task is the low bending stiffness of the rope. Standard linear beam finite element assumes the independence of tension-compression and bending, which is wrong for problems of bending of a preliminary stretched string with low bending stiffness. The moments and transverse shear forces arising from the stretching were simulated, on the one hand, by projections of the tensile cable forces onto the previously bent axis of the rope and, on the other hand, by the springs that rotate and are fixed to the immovable space.

The calculation of the minimum pitch of laying, taking into account the elasticity of the lining, was carried out for four values of the depth of the groove $(0.3 ; 0.33 ; 0.40$ and 0.45 of the rope diameter) and for seven values of the drum diameters $(60,80, \ldots, 180$ rope diameters). The results are presented in Figure 9 as recommendations for a rational rope winding pitch. The contact stresses between the individual wire of the rope and the elastic liner made of phenolcapron material are determined by the average pressure of the rope on the liner at the site and along the length of this section, taking into account the wire-strand structure and the stripe of the rope. For the selected profile of the scallop, this design scheme allows us to estimate the rigidity, taking into account the influence of the free lining areas. 


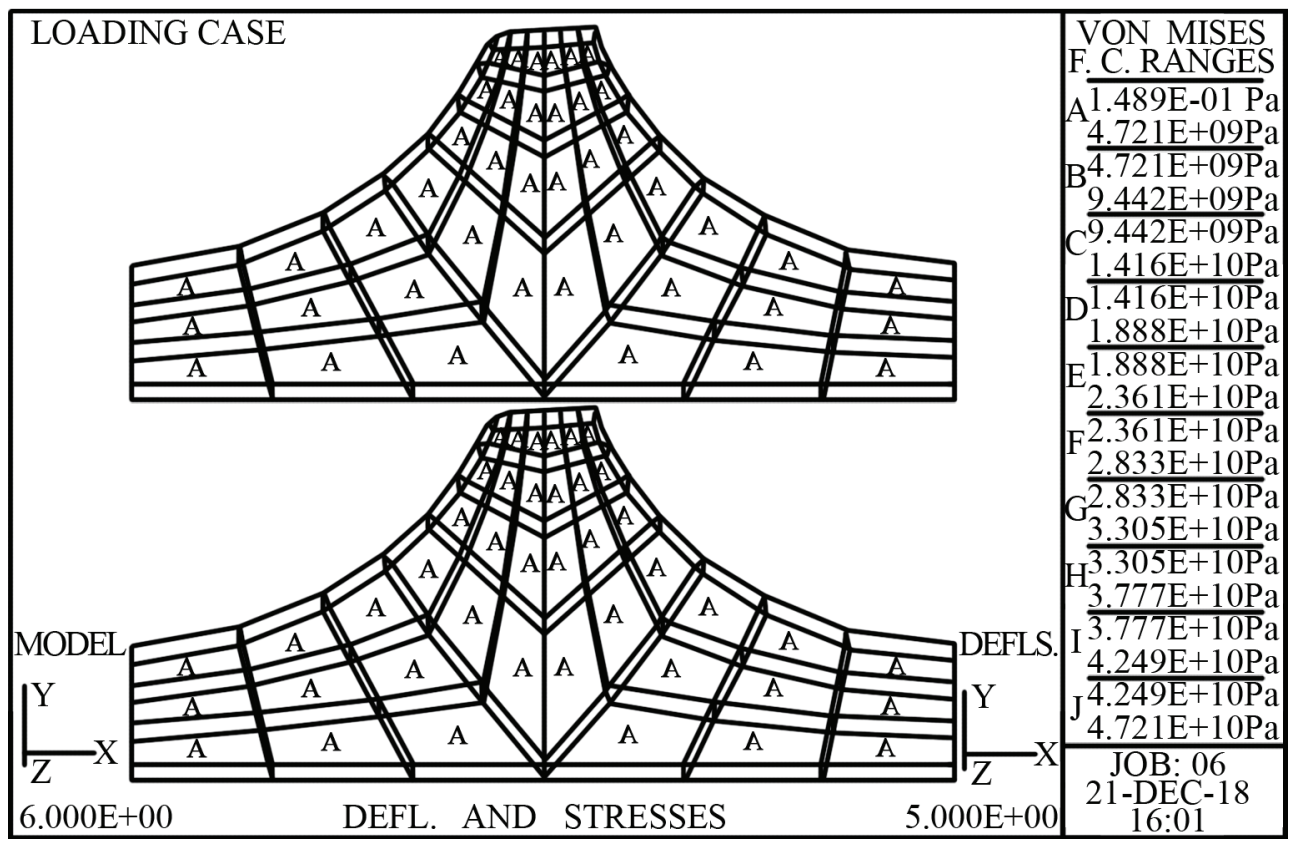

Fig. 11. Stress-strain state in two parts of the scallop (screen form).

Figure 12 shows the graphical dependence of the laying pitch on the diameters of the drum and the rope, as well as the depth of the groove: the solid line is the phenolcapron lining, the dotted line is the non-lined drum.

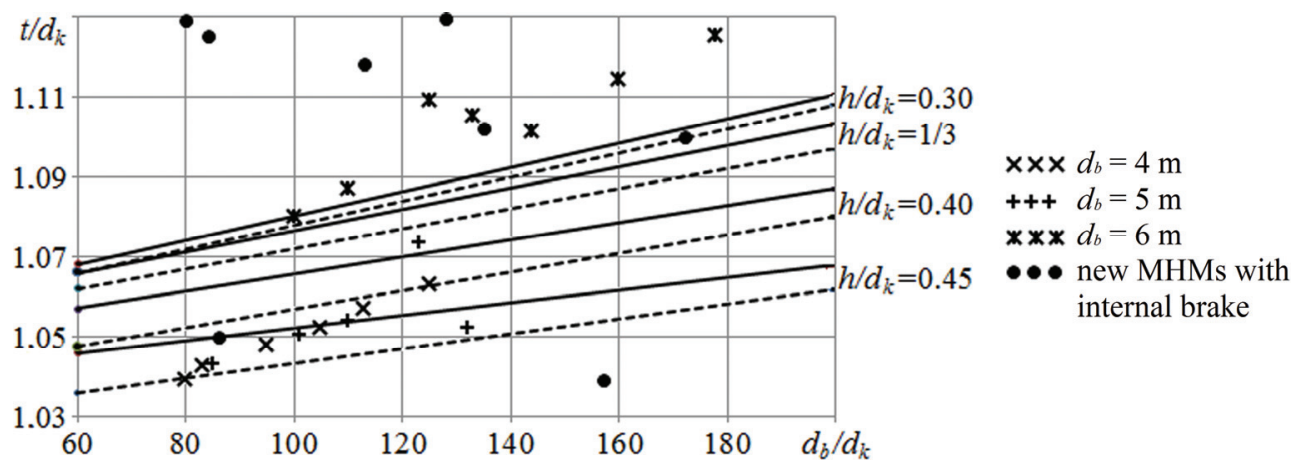

Fig. 12. The dependence of the pitch of laying on the diameters of the drum and rope.

\section{Conclusions}

The problem of finding the minimum distance between the adjacent turns of the rope during winding depending on the ratio of the diameters of the drum and the rope, the angle of deviation and the pitch of winding are considered. Several models of interaction between the rope and the shell, which differ in the laboriousness of the calculation and the degree of account of the elastic properties of the rope and the lined or non-lined shell are proposed. It is established that each transition to a more complex model entails an increase in the groove cutting pitch by $0.2-0.5 \%$. This is explained by the fact that each following model differs from the previous one in view of the compliance of sections that were previously assumed to be absolutely rigid. At the same time, even the simplest geometric theory of winding 
requires solving nonlinear equations with moving boundary conditions and a large expenditure of computer time, which necessitates the use of developed models implemented in the form of programs.

As it follows from the analysis, formula (1) gives an underestimation of the laying pitch for 4- and 5-meter MHMs by $3 \%$. Formula (3) for ropes of large diameter and shallow grooves gives a match with our curves and for ropes with smaller diameters overestimation of the pitch by $2.5 \%$.

When choosing a tilda-profile, the required cutting pitch is overestimated by $5 \%$.

It is recommended to use the U-tilda-profile, which will allow a $4.5 \%$ increase in the cable capacity while maintaining the radius of curvature of the rope, which is admissible according to the safety rules, during the installation process.

Deepening the groove by $5 \%$ in the range from 0.3 to 0.45 rope diameters reduces the required groove pitch by an average of $1-1.5 \%$. So, if the diameter of the drum of MHM is 100 times the diameter of the rope, then by deepening the groove from 0.3 to 0.45 of the diameter of the rope, one can reduce the required cutting pitch from 1.08 to 1.052 diameters of the rope.

Increasing the ratio of the diameter of the drum of MHM to the diameter of the rope from 80 to 180 increases the required cutting pitch from $3 \%$ for a groove with a depth of 0.3 for the diameter of the rope to $2 \%$ for a deeper one ( 0.45 diameters).

Studies of the spatial interaction of the rope with an elastic lining showed that, due to the increase in the arc of the scallop of the neck, the contact pressure (taking into account the wire-strand structure of the rope) decreases and does not exceed the lining allowed for this material, such as phenolcapron. The use of such a lining causes an increase in the pitch of cutting the groove by no more than $1 \%$.

\section{References}

1. Zabolotny, K., Zinovyev, S., Zupiev, A., Panchenko, E. (2015). Rationale for the parameters equipment for rope dehydration of mining hoisting installations, New Developments in Mining Engineering 2015: Theoretical and Practical Solutions of Mineral Resources Mining, 275-283

2. Zabolotnyi, K.S., Zhupiiev, O.L. (2012). Study of deflected mode of mine winder split drums, Scientific bulletin of the National Mining University, 1, 82-88

3. Kiselev, N.N., Kuzhel, M.G., Dimashko, A.D., Ilin, P.L. (1954). K voprosu o dopuskayemom shage navivki kanata na baraban podyemnoy mashiny, Ugol, 11, 27-31

4. Dimashko, A.D., Gershikov, I.YA., Krenevich, A.A. (1973). Shakhtnyye elektricheskiye lebedki i podyemnyye mashiny: Spravochnik. Moskva: Nedra

5. Zabolotnyi, K.S. (1997). Razrabotka ratsionalnykh parametrov barabanov shakhtnykh podyemnykh mashin s vnutrennimi tormozami na osnove metodov chislennogo modelirovaniya. Dnepropetrovsk: DNVP «Sistemnyye tekhnologii» 